\title{
On computing residue integrals for a class of nonlinear transcendental systems
}

\author{
A. A. Kytmanov, A. M. Kytmanov, E. K. Myshkina \\ Siberian Federal University \\ Krasnoyarsk, 660041, Russia \\ aakytm@gmail.com, akytmanov@sfu-kras.ru, elfifenok@mail.ru
}

\begin{abstract}
A method of computing residue integrals with integration over certain cycles for systems of transcendental equations is presented. Such integrals are connected to the power sums of roots for a certain system of equations. The described approach can be used for developing methods for the elimination of unknowns from transcendental systems.
\end{abstract}

The problem of elimination of unknowns from systems of nonlinear algebraic equations is a classical algebraic problem. The elimination method based on the notion of a Gröbner basis and suggested by B. Buchberger is one of the main elimination methods in polynomial computer algebra nowadays (see, e.g., [4], [1]). However, this method is inapplicable to non-polynomial systems.

In 1970s in [2] L. A. Aizenberg proposed a new elimination method based on the multidimensional residue theory, namely on the formulas of multidimensional logarithmic residue and Grothendieck residue. The basic idea of the method was to find certain residue integrals connected to the power sums of roots of a given system of equations without finding the roots themselves. Its further developments were implemented in [3], [13], and [6].

In applied problems of chemical kinetics, systems of transcendental equations, namely, systems consisting of exponential polynomials [5], [8] (Zeldovich-Semenov model, etc.), arise as well. However, the elimination method developed in [3], [13], [6] can not be applied to this kind of systems. One of the obstacles is the fact that the set of roots of a system of $n$ transcendental equations in $n$ variables is, in general, infinite. Moreover, multi-Newton sums (with powers in $\mathbb{N}^{n}$ ) of the roots of such systems lead usually to divergent series.

In the present work we compute residue integrals for a specific kind of systems of $n$ transcendental equations, and deduce from this computation (provided such series converge) the values of the sums of multi-Newton series consisting of the roots of such systems which do not belong to coordinate subspaces. In other words, we generalize the statements from [12], [7], [9], [11], [10] to a wider class of systems of transcendental equations.

We consider a system of functions $f_{1}(z), \ldots, f_{n}(z)$ of the form

$$
f_{i}\left(z_{1}, \ldots, z_{n}\right)=q_{i}\left(z_{1}, \ldots, z_{n}\right)+Q_{i}\left(z_{1}, \ldots, z_{n}\right), \quad i=1, \ldots, n,
$$

where

$$
q_{i}\left(z_{1}, \ldots, z_{n}\right)=\left(1-a_{i 1} z_{1}\right)^{m_{i 1}} \cdot \ldots \cdot\left(1-a_{i n} z_{n}\right)^{m_{i n}}, \quad i=1, \ldots, n,
$$

$m_{i j}$ are positive integers, $a_{i j} \in \mathbb{C} \backslash\{0\}$, and $Q_{i}(z)$ are entire functions for $i=1, \ldots, n$.

Let $J=\left(j_{1}, \ldots, j_{n}\right)$ be a multi-index where $\left(j_{1} \ldots j_{n}\right)$ is a permutation of $(1 \ldots n)$, and let $a_{J}=$ $\left(a_{1 j_{1}}, \ldots, a_{n j_{n}}\right)$. 
The system

$$
q_{i}(z)=0, \quad i=1, \ldots, n
$$

has $n$ ! isolated roots in $\mathbb{C}^{n}$. The roots of (1) are multi-indices

$$
\tilde{a}_{J}=\left(1 / a_{1 j_{1}}, \ldots, 1 / a_{n j_{n}}\right) .
$$

By $\Gamma_{q}$ we denote the cycle

$$
\Gamma_{q}=\left\{z \in \mathbb{C}^{n}:\left|q_{i}(z)\right|=r_{i}, r_{i}>0, i=1, \ldots, n\right\} .
$$

Consider the system of equations

$$
F_{i}(z, t)=q_{i}(z)+t \cdot Q_{i}(z)=0, \quad i=1, \ldots, n,
$$

which depends on a real parameter $t \geqslant 0$.

Let $r_{1}>0, \ldots, r_{n}>0$ be fixed real numbers. Then, for sufficiently small $t>0$, the inequalities

$$
\left|q_{i}(z)\right|>\left|t \cdot Q_{i}(z)\right|, i=1, \ldots, n
$$

hold on the cycles $\Gamma_{q}$ which is due to compactness of $\Gamma_{q}$.

By $J_{\gamma}(t)$ we denote the residue integral

$$
J_{\gamma}(t)=\frac{1}{(2 \pi \sqrt{-1})^{n}} \int_{\Gamma_{q}} \frac{1}{z^{\gamma+1}} \cdot \frac{d F}{F}=\frac{1}{(2 \pi \sqrt{-1})^{n}} \int_{\Gamma_{q}} \frac{1}{z_{1}^{\gamma_{1}+1} \cdot \ldots \cdot z_{n}^{\gamma_{n}+1}} \cdot \frac{d F_{1}}{F_{1}} \wedge \ldots \wedge \frac{d F_{n}}{F_{n}},
$$

where $\gamma=\left(\gamma_{1}, \ldots \gamma_{n}\right)$ is a multi-index and $I=(1, \ldots, 1)$.

In order to formulate the main result, we introduce the following notations.

Denote by $\Delta=\Delta(t)$ the Jacobian of the system $F_{1}(z, t), \ldots, F_{n}(z, t)$ with respect to $z_{1}, \ldots, z_{n}$. Let $(-1)^{s(J)}$ be the sign of the permutation $J$, and $\alpha=\left(\alpha_{1}, \ldots, \alpha_{n}\right)$ be a multi-index of length $n$. By $q^{\alpha+I}(J)$ we denote $q_{1}^{\alpha_{1}+1}\left[j_{1}\right] \cdot \ldots \cdot q_{n}^{\alpha_{n}+1}\left[j_{n}\right]$, where $q_{s}\left[j_{s}\right]$ is a product of all $\left(1-a_{j 1} z_{1}\right)^{m_{j 1}} \cdot \ldots \cdot\left(1-a_{j n} z_{n}\right)^{m_{j n}}$ except $\left(1-a_{s j_{s}} z_{s}\right)^{m_{s j_{s}}}$. Also by $\beta(\alpha, J)$ we denote the vector $\left(m_{1 j_{1}}\left(\alpha_{j_{1}}+1\right)-1, \ldots, m_{n j_{n}}\left(\alpha_{j_{n}}+1\right)-1\right)$, and $\beta(\alpha, J) !=\prod_{p}\left(m_{p j_{p}}\left(\alpha_{j_{p}}+1\right)-1\right)$ !. Finally, $a_{j}^{\beta+I}$ denotes $a_{1 j_{1}}^{m_{1 j_{1}}\left(\alpha_{j_{1}}+1\right)} \cdot \ldots \cdot a_{n j_{n}}^{m_{n j_{n}}\left(\alpha_{j_{n}}+1\right)}$, and

$$
\frac{\partial^{\| \beta(\alpha(J) \|}}{\partial z^{\beta(\alpha, J)}}=\frac{\partial^{m_{1 j_{1}}\left(\alpha_{j_{1}}+1\right)-1+\ldots+m_{n j_{n}}\left(\alpha_{j_{n}}+1\right)-1}}{\partial z_{1}^{m_{1 j_{1}}\left(\alpha_{j_{1}}+1\right)-1} \ldots \partial z_{n j_{n}\left(\alpha_{j_{n}}+1\right)-1}^{m_{n_{1}}}} .
$$

Theorem 1 Under the assumptions made for the functions $F_{i}$ defined by (2) the following formulas for $J_{\gamma}(t)$ as convergent (for sufficiently small $t$ ) series are valid:

$$
J_{\gamma}(t)=\sum_{J} \sum_{\alpha}(-t)^{\|\alpha\|+\| \beta(\alpha, J) \mid+n} \frac{(-1)^{s(J)}}{\beta(\alpha, J) ! \cdot a_{J}^{\beta+I}} \cdot \frac{\partial^{\| \beta(\alpha(J) \|}}{\partial z^{\beta(\alpha, J)}}\left[\frac{\Delta(t)}{z_{1}^{\gamma_{1}+1} \cdot \ldots \cdot z_{n}^{\gamma_{n}+1}} \cdot \frac{Q^{\alpha}}{q^{\alpha+I}(J)}\right]_{z=\tilde{a}_{J}},
$$

where the summation is over all the multi-indices $J$.

These residue integrals are closely connected to the power sums of roots of the corresponding systems of equations and therefore are essential for the development of elimination methods.

This research was supported by grant of the President of the Russian Federation for young scientists, project MD-197.2017.1, by grant of the Government of the Russian Federation for investigations under the guidance of the leading scientists of the Siberian Federal University (contract No. 14.Y26.31.0006), and by the Russian Foundation for Basic Research, project 15-01-00277-a. 


\section{References}

[1] Adams, W.W., Loustaunau, P. An introduction to Gröbner bases. Graduate Studies in Mathematics, v. 3. Amer. Math. Soc., Providence, RI, 1994.

[2] Aizenberg, L.A. On a formula of the generalized multidimensional logarithmic residue and the solution of system of nonlinear equations. Sov. Math. Doc, 18:691-695, 1977.

[3] Aizenberg, L.A., Yuzhakov, A.P. Integral representations and residues in multidimensional complex analysis. Trans. Amer. Math. Monographs, AMS, Providence, 1983.

[4] Buchberger, B. Gröbner bases, an algorithmic method in polynomial ideal theory. in Multidimensional Systems Theory (N. K. Bose ed.), Reidel, Dordrecht, 184-232, 1985.

[5] Bykov, V.I. Modeling of the critical phenomena in chemical kinetics. Komkniga, Moscow (in Russian), 2006.

[6] Bykov, V., Kytmanov, A., Lazman, M., Passare, M. (ed) Elimination Methods in Polynomial Computer Algebra. Math. and Appl., v. 448, Kluwer Acad. Publ., Dordreht, Boston, London, 1998.

[7] Bykov, V.I., Kytmanov, A.M., Myslivets, S.G. Power sums of nonlinear systems of equations. Docl. Math., 76(2):641-645, 2007.

[8] Bykov, V.I., Tsybenova, S.B. Non-linear models of chemical kinetics, KRASAND, Moscow (in Russian), 2011.

[9] Kytmanov, A.A. Analogs of Recurrent Newton Formulas. Russian Mathematics, 53 (10):34-44, 2009.

[10] Kytmanov, A.A., Kytmanov, A.M., Myshkina, E.K. Finding Residue Integrals for Systems of Nonalgebraic Equations in $\mathbb{C}^{n}$. Journal of Symbolic Computation, 66:98-110, 2015.

[11] Kytmanov, A.M., Myshkina, E.K. Evaluation of power sums of roots for systems of non-algebraic equations in $\mathbb{C}^{n}$. Russian Mathematics 57(12):31-43, 2013.

[12] Kytmanov, A.M., Potapova, Z.E. Formulas for determining power sums of roots of systems of meromorphic functions. Izvestiya VUZ. Matematika, 49 (8):36-45, 2005 (in Russian).

[13] Tsikh, A.K. Multidimensional residues and their applications. Translations of Mathematical Monographs, 103. American Mathematical Society, Providence, RI., 1992. 